\title{
How to Make a Strongly Connected Digraph Two-Connected
}

\author{
A. Frank ${ }^{1}$ and T. Jordán ${ }^{2} 1$ \\ 1 Department of Computer Science, Eötvös University, 1088 Budapest, Hungary, \\ 2 CWI, Kruislaan 413, 1098 SJ, Amsterdam, The Netherlands
}

\begin{abstract}
Given a strongly connected digraph, we give a combinatorial polynomial algorithm for determining a smallest set of new edges to be added to make the graph 2-vertex-connected. The problem was shown to be polynomially solvable in a recent paper [FJ1] for arbitrary starting digraph and any target connectivity $k \geq 1$. However, the algorithm relied on the ellipsoid method. Here we further simplify the results of [FJ1] and [Jor3] by some structural statements related to families of ordered pairs of subsets.
\end{abstract}

\section{Introduction}

This paper deals with the directed vertex-connectivity augmentation problem: given a digraph $G$, the goal is to find a smallest set of new edges which makes $G k$-vertex-connected.

The first proof for the polynomial solvability of this problem appears in [FJ1]. However, the algorithm still uses the ellipsoid method, and developing a purely combinatorial polynomial algorithm is an exciting open problem. Before this general result, only the special case $k=1$ was solved, see [ET1] for a linear-time algorithm.

The main purpose of this paper is to describe a combinatorial algorithm for finding an optimal augmenting set which makes a strongly connected digraph two-connected. This special case was also open and requires completely new methods, as well.

The key of the algorithm is a lemma on the existence of saturating edges, i.e. edges which are contained in some optimal augmentation. This lemma is strongly related to a general splitting theorem for directed vertex-connectivity. The latter claims that in a $k$-connected digraph $G$, if some conditions hold, two edges $t s$ and $s u$ can be replaced by a new edge $t u$, without destroying $k$-connectivity. In Section 3 we obtain an augmentation algorithm from this result, which is purely combinatorial, and gives the optimal solution in our special case.

In Section 4 the general min-max theorem of [FJ1] will be simplified with the help of the results of Section 2. These results describe the structure of an

\footnotetext{
${ }^{1}$ Research was partially supported by the Hungarian National Foundation for Scientific
} Research, grants OTKA 2118, F014919. 
independent family of pairs of subsets. This yields another proof (and a similar algorithm) for the two-connectivity augmentation results of the previous section. (And for any case where the target connectivity is "small" and one bigger than the connectivity of the starting digraph.)

Note, that the corresponding undirected $k$-connectivity augmentation problem is still not solved, even if the starting graph is $(k-1)$-connected. A polynomial algorithm is known only for the small cases $k \leq 3$, see [ET1],[WN2] and [HR1]. For arbitrary $k$, an "almost" min-max equality is given in [Jor1], see also [Jor2]. The situation is much better for edge-connectivity. The general problem - directed and undirected - can be solved in polynomial time, and there are nice combinatorial algorithms, see [WN1],[Fra1] and [NGM1]. For a survey on augmentations, see [Fra2].

\section{Independent Families of Ordered Pairs of Subsets}

Given a finite ground-set $S$, by a pair we always mean an ordered pair of disjoint non-empty subsets of $S$. We will use the notation $(T, F)$ for a pair with tail $T$ and head $F$. Two pairs $\left(T_{1}, F_{1}\right)$ and $\left(T_{2}, F_{2}\right)$ are said to be independent, if

$$
T_{1} \cap T_{2}=\emptyset \text { or } F_{1} \cap F_{2}=\emptyset
$$

A family $\mathcal{F}$ of pairs is independent, if the members of $\mathcal{F}$ are pairwise independent.

Let $n:=|S|$. For convenience, we will use the notation $C:=S-(T \cup F)$ for a pair $(T, F)$, when the pair is clear from the context, for example we will say that $C_{i}$ belongs to $\left(T_{i}, F_{i}\right)$.

We will study the properties of the independent families of pairs. In a family $\mathcal{F}$ the pairs which include a fixed vertex $v \in S$ in their tails have pairwise disjoint heads. This gives immediately an upper bound $|\mathcal{F}| \leq n(n-1)$ for the size of an independent family. This bound is sharp, see the family $\{(v, w): v, w \in S, v \neq$ $w\}$.

Let us restrict our attention to families of a special type. We say that a family $\mathcal{F}$ of pairs on $n$ vertices is $k$-separated for some $0 \leq k \leq n-2$ if the following three conditions hold:

$$
|F \cup T|=n-k \text { for every }(T, F) \in \mathcal{F}
$$

and for any two pairs $(T, F),\left(T^{\prime}, F^{\prime}\right) \in \mathcal{F}$

$$
\begin{aligned}
& \left|T \cup T^{\prime}\right| \leq n-k-\left|F \cap F^{\prime}\right| \text { if } F \cap F^{\prime} \neq \emptyset \\
& \left|F \cup F^{\prime}\right| \leq n-k-\left|T \cap T^{\prime}\right| \text { if } T \cap T^{\prime} \neq \emptyset
\end{aligned}
$$

We say that a head or tail $X$ of some pair in a $k$-separated family is small, if $|X| \leq\lfloor(n-k) / 2\rfloor$ holds. 
In the following lemmas let $\mathcal{F}$ be a $k$-separated independent family of pairs.

Lemma 2.1. If $\left|F_{1}\right| \geq\left|T_{2}\right|$ for two pairs $\left(T_{1}, F_{1}\right)$ and $\left(T_{2}, F_{2}\right)$, then $T_{1} \cap T_{2}=\emptyset$. Analogously, if $\left|T_{1}\right| \geq\left|F_{2}\right|$, then $F_{1} \cap F_{2}=\emptyset$.

Proof. By symmetry, it is enough to prove the first assertion. For suppose $\left|F_{1}\right| \geq$ $\left|T_{2}\right|$, but the pairs are not tail-disjoint. Thus $F_{1} \cap F_{2}=\emptyset$ since $\mathcal{F}$ is independent. From the axioms we get $k=\left|T_{1} \cap C_{2}\right|+\left|C_{1} \cap C_{2}\right|+\left|F_{1} \cap C_{2}\right|$ and $\left|T_{1} \cap C_{2}\right|+\mid C_{1} \cap$ $C_{2}|+| T_{2} \cap C_{1} \mid \geq k$. This gives $\left|T_{2} \cap C_{1}\right| \geq\left|F_{1} \cap C_{2}\right|$, which yields $\left|T_{2}\right|>\left|F_{1}\right|$, a contradiction.

Lemma 2.2. Let $F_{1} \cap F_{2} \neq \emptyset$. Then $\left|S-\left(F_{1} \cup F_{2}\right)\right| \leq k$.

Proof. By the axioms $2 k=\left|C_{1}\right|+\left|C_{2}\right|=\left|C_{1} \cap F_{2}\right|+\left|C_{2} \cap F_{1}\right|+\left|C_{1} \cap C_{2}\right|+\mid S-$ $\left(F_{1} \cup F_{2}\right)|\geq k+| S-\left(F_{1} \cup F_{2}\right) \mid$, which proves the lemma.

The following lemma is straightforward from Lemma 2.1:

Lemma 2.3. The small heads in $\mathcal{F}$ are pairwise disjoint, and the small tails in $\mathcal{F}$ are pairwise disjoint.

A bipartition of $\mathcal{F}$ is a partition of $\mathcal{F}$ into two disjoint parts, such that one part consists of pairwise head-disjoint pairs, and the other part consists of pairwise tail-disjoint pairs.

We define the canonical bipartition $S_{t} \cup S_{f}$ of $\mathcal{F}$ as follows. Let $S_{f}$ contain the pairs with small heads and let the remaining pairs belong to $S_{t}$. By Lemma 2.3 , this is indeed a bipartition of the family.

We say that a pair is free subject to a given bipartition, if putting it to the other part, the new parts form a bipartition again. (That is, if the pair is in the tail-disjoint part, then it is head-disjoint from every pair in the head-disjoint part, or conversely.)

Lemma 2.4. Every bipartition $A_{t} \cup A_{f}$ has a free pair.

Proof. Let us consider the tails in $A_{t}$ and the heads in $A_{f}$. Choose a set of maximum size from these sets. We can assume it is a tail $T^{\prime}$. We claim that the pair $\left(T^{\prime}, F^{\prime}\right) \in A_{t}$ is free. Indeed, $\left|T^{\prime}\right| \geq|F|$ holds for every pair $(T, F) \in A_{f}$ by the choice of $T^{\prime}$, and $F^{\prime} \cap F=\emptyset$ by Lemma 2.1 .

The following theorem simplifies the structure of an independent family $\mathcal{F}$ if the number of pairs is high enough.

Theorem 2.5. Suppose that $|\mathcal{F}| \geq 2 k+2$. Then the heads are pairwise disjoint or the tails are pairwise disjoint. 
Proof. First observe that there are no two heads (or tails) such that one is included in the other. For suppose $F_{1} \subseteq F_{2}$. Thus $T_{2} \subset C_{1}$ holds, which is impossible, since $\left|C_{1}\right|=k$ and $\left(S-\left(T_{1} \cup T_{2}\right)-\left(F_{1} \cap F_{2}\right)\right) \subset C_{1}$.

The next claim is that there exist $k+2$ pairs in $\mathcal{F}$ which are pairwise headdisjoint or pairwise tail-disjoint. Indeed, the bigger part of the canonical bipartition will do, except when $\left|S_{t}\right|=\left|S_{f}\right|=k+1$. However, there exists a free pair by Lemma 2.4 , which proves that we can find $k+2$ pairwise head- or tail-disjoint pairs.

Thus we have $k+2$ pairs $\left(T_{1}, F_{1}\right), \ldots,\left(T_{k+2}, F_{k+2}\right)$ with pairwise disjoint say - heads. Add further pairs to this sub-family until it is possible to maintain the disjointness of the heads. Suppose that there is a pair $(T, F)$ which can not be added. Let - without loss of generality $-F \cap F_{1} \neq \emptyset$. By Lemma 2.2 this implies $\left|S-\left(F \cup F_{1}\right)\right| \leq k$. But this yields that the head of some pair of the current subfamily must be a subset of $F$, a contradiction.

The following easy observation connects the previous results to the vertexconnectivity augmentation problem of digraphs.

Recall, that a digraph $G=(V, E)$ is $k$-connected, if $|V| \geq k+1$ and $G-X$ is strongly connected for any subset $X \subset V$ with $|X| \leq k-1$. We say that a pair $(T, F), \emptyset \neq T, F \subset V, T \cap F=\emptyset$ is a one-way pair if there is no edge in $G$ with tail in $T$ and head in $F$. For a one-way pair $(T, F)$ let $h(T, F):=|V-(T \cup F)|$. Observe, that by Menger's Theorem $h(T, F) \geq k$ for every one-way pair if and only if $G$ is $k$-connected.

Lemma 2.6. Let $G=(V, E)$ be a $k$-connected directed graph. Let $\mathcal{F}$ consist of the one-way pairs $(T, F)$ of subsets of $V$ for which $h(T, F)=k$. Then $\mathcal{F}$ is a $k$-separated family of pairs.

Thus we say that a one-way pair $(T, F)$ with $h(T, F)=k$ is $k$-separated.

\section{Optimal Two-Connectivity Augmentation of Digraphs}

This section contains a solution to the optimal two-connectivity augmentation problem in digraphs. First we state some lemmas which are valid for any $k$.

Let $\Gamma^{+}(X)$ and $\Gamma^{-}(X)$ denote the set of out-neighbours and the set of inneighbours of a set $X$ of vertices, respectively. We say that a set $X \subset V$ with $|V-X| \geq k+1$ is out-tight in a $k$-connected digraph $G=(V, E)$ if $\left|\Gamma^{+}(X)\right|=$ $k$. Similarly, $X$ is in-tight if $\left|\Gamma^{-}(X)\right|=k$ and $|V-X| \geq k+1$ hold. The maximum number of pairwise disjoint in-tight and out-tight sets in $G$ is denoted by $b(G)$ and $t(G)$, respectively. For an out-tight set $X$ let $X^{*}$ denote the set $V-X-\Gamma^{+}(X)$. If $X$ is in-tight, $X^{*}:=V-X-\Gamma^{-}(X)$. Observe that if $X$ is in-tight, $X^{*}$ is out-tight. Furthermore, the set of pairs $\left(X, X^{*}\right)$, where $X$ is out-tight, is precisely the same as the set of $k$-separated one-way pairs of $G$.

The well-known submodular property of $\left|\Gamma^{+}\right|$and $\left|\Gamma^{-}\right|$implies: 
Lemma 3.1. Let $X$ and $Y$ be two in-tight (out-tight) sets in a k-connected digraph $G=(V, E)$ with $X \cap Y \neq \emptyset$ and $|V-(X \cup Y)| \geq k$. Then $X \cap Y$ is in-tight (out-tight). Moreover, if $|V-(X \cup Y)| \geq k+1$ holds, then $X \cup Y$ is also in-tight (out-tight).

Observe, that the second part of the lemma states that if $T_{1} \cap T_{2} \neq \emptyset$ and $F_{1} \cap F_{2} \neq \emptyset$ for two $k$-separated one-way pairs $\left(T_{1}, F_{1}\right)$ and $\left(T_{2}, F_{2}\right)$, then $\left(T_{1} \cap\right.$ $\left.T_{2}, F_{1} \cup F_{2}\right)$ and $\left(T_{1} \cup T_{2}, F_{1} \cap F_{2}\right)$ are $k$-separated one-way pairs, as well.

Lemma 3.2. Let $b(G) \geq k+1 \quad(t(G) \geq k+1)$ in a $k$-connected digraph $G$. Then the minimal in-tight (out-tight) sets are pairwise disjoint.

Proof. Let $\mathcal{B}$ be a maximal size family of pairwise disjoint minimal in-tight sets. Hence $|\mathcal{B}| \geq k+1$. We claim that $X \in \mathcal{B}$ for every minimal in-tight set $X$. If this is not the case, $X$ and some $Y \in \mathcal{B}$ are intersecting. Since $X$ can not contain any member of $\mathcal{B},|V-(X \cup Y)| \geq k$. Then $X \cap Y$ would be in-tight by Lemma 3.1 , a contradiction.

Let $m_{k}(G)$ or simply $m(G)$ denote the minimum number of new edges to be added to make $G(k+1)$-connected. An edge $e$ is saturating, if $\max \{b(G+e), t(G+$ $e)\} \leq \max \{b(G), t(G)\}-1$. (We use the notation $G+e$ for $(V(G), E(G) \cup\{e\})$.) For a minimal in-tight set $B$, let $B_{c}$ denote the container-set of $B$, i.e. the union of those in-tight sets which contain $B$ but disjoint from the other minimal intight sets. The container-set of a minimal out-tight set is defined analogously.

Lemma 3.3. Let $B$ be a minimal in-tight set in a $k$-connected digraph $G$ with $b(G) \geq k+1$. Let $\left|V-B_{c}\right| \geq k+1$ and let $x \in B$ and $y \in B_{c}^{*}$. Then $b(G+(y x))=$ $b(G)-1$.

Proof. Let $\mathcal{B}$ and $\mathcal{B}^{\prime}$ be the families of minimal in-tight sets of $G$ and $G^{\prime}:=$ $G+(y x)$, respectively. If $b\left(G^{\prime}\right)=b(G)$, then all the members of $\mathcal{B}-B$ are in $\mathcal{B}^{\prime}$, too, and $\mathcal{B}^{\prime}$ contains an in-tight set $B^{\prime}$ for which $B \subset B^{\prime}$. Since $B^{\prime}$ is in-tight in $G, B^{\prime} \subset B_{c}$ follows, a contradiction.

If we could always find a saturating edge, the min-max theorem $m(G)=$ $\max \{b(G), t(G)\}$ would follow. However, this is not the case, as the examples of [Jor3] show for any $k \geq 1$. (But if $m(G)$ is large enough, this equality holds. We will prove it for $k=2$ directly and deduce from the general min-max equality for any $k$.)

Lemma 3.4. Let $k+2 \leq \max \{b(G), t(G)\}=b(G)$ for a $k$-connected digraph and let $v \in B$ be a vertex in a minimal in-tight set $B$. Then there exists a saturating edge with head $v$.

Proof. Since every container-set $B_{c}$ is a union of in-tight sets, and now $\left|V-B_{c}\right| \geq$ $k+1$, it follows that $B_{c}$ is itself in-tight and there exists an edge $e$ by Lemma 
3.3 for which $b(G+e)=b(G)-1$. Thus we can assume that $b(G)=t(G)$. In this case we get similarly that there exists an edge $f$ for which $t(G+f)=t(G)-1$. We have to prove that there exists a common edge $e=f$.

For this we need a minimal in-tight set $B$ and a minimal out-tight set $K$ for which

$$
B_{c}^{*} \cap K \neq \emptyset \text { and } K_{c}^{*} \cap B \neq \emptyset
$$

It is easy to see that this is equivalent to the condition that $\left(B_{c}^{*}, B_{c}\right)$ and $\left(K_{c}, K_{c}^{*}\right)$ are not independent pairs. Indeed, (3.1) implies that they are not independent. Conversely, if they are not independent, then $K_{c} \cap B_{c}^{*}$ is out-tight and $B_{c} \cap K_{c}^{*}$ is in-tight. By definition, the container-sets contain only one minimal tight set, thus $K \subseteq B_{c}^{*}$ and $B \subseteq K_{c}^{*}$ follows. (Note, that this implies that if there exists a saturating edge between $K$ and $B$ then any edge between $K$ and $B$ is saturating.)

Let $B$ be a fixed minimal in-tight set, and $K$ an arbitrary minimal out-tight set in $B_{c}^{*}$. If (3.1) does not hold for $B$ and $K$, then $\left|V-\left(B_{c}^{*} \cup K_{c}\right)\right| \leq k$ by Lemma 2.2. Since the out-tight container-sets are pairwise disjoint, there exists a minimal out-tight set $L$ for which $L_{c} \subset B_{c}^{*}$ holds. Now $B$ and $L$ satisfy (3.1).

The following splitting result of [Jor3] implies Lemma 3.4, as one can prove easily. Here we sketch a simple proof of that result and show that these two statements are strongly related.

A pair $t s$ and $s u$ of edges in a $k$-connected digraph $G$ is said to be splittable from $s$ if the graph remains $k$-connected after replacing these two edges by a new edge $t u$. An edge $e$ is critical - with respect to $k$-connectivity - if $G-e$ is not $k$-connected.

Theorem 3.5. [Jor3] Let $G=(V, A)$ be a $k$-connected directed graph, and let $\left|\Gamma^{+}(s)\right| \geq k+1$ and $\left|\Gamma^{-}(s)\right| \geq k+1$ for some $s \in V$. Then every critical edge ts (or su) is in a splittable pair (from $s$ ).

Proof. The idea is that there exists a saturating edge even if we weaken the conditions of the previous lemma a little bit. The graph $G-s$ is $(k-1)$-connected. For an out-neighbour $v$ of $s$ let us define $B_{v}$ to be the maximal in-tight set - with respect to $(k-1)$-connectivity - in $G-s$ which contains no other neighbours of $s$, and if there is no such set, let $B_{v}=\{v\}$. In this latter case let $B_{v}^{*}:=V-B_{v}$. Similarly for the in-neighbours of $s$. Observe that a pair $t s$ and $s u$ is splittable if and only if (3.1) holds for $K_{t}$ and $B_{u}$. The existence of a splittable pair which contains ts follows the same way as the existence of the saturating edge in Lemma 3.4 .

Lemma 3.6. If $b(G) \geq 2 k+1, t(G) \geq k+2$ and $b(G) \geq t(G)$ for a $k$-connected digraph $G$ then the minimal out-tight sets can be matched into a subset of minimal in-tight sets such that every pair $B_{i}, K_{i}$ satisfies $(3.1), i=1, \ldots, t(G)$.

Proof. We saw that (3.1) holds if and only if the two pairs $\left(B_{c}^{*}, B_{c}\right)$ and $\left(K_{c}, K_{c}^{*}\right)$ are not independent. If we assign blue and red vertices to the minimal in-tight 
and out-tight sets, respectively, and connect two vertices of different colour if and only if (3.1) holds for the corresponding minimal tight sets, then the question is whether there exists a matching of size $t(G)$ in this bipartite graph or not.

Suppose that the red vertices are not matchable into the blue colour-class. Then by Hall's Theorem there is a subset $\mathcal{B}$ of the minimal in-tight sets and a subset $\mathcal{K}$ of the minimal out-tight sets such that (3.1) does not hold for any pair $B, K$, where $B \in \mathcal{B}, K \in \mathcal{K}$ and $|\mathcal{B}|+|\mathcal{K}|=: r>b(G) \geq 2 k+1$. Thus, by Theorem 2.5, we can find $r>b(G) \geq t(G)$ pairwise disjoint heads (or tails) in $\mathcal{B} \cup \mathcal{K}$, a contradiction.

Note, that the addition of the set of edges of such a pairing (oriented from $K_{i}$ to $\left.B_{i}\right)$ not necessarily makes the graph $(k+1)$-connected, even if $b(G)=t(G)$. (However, as we will see in Theorem 4.2, in that case there exists a pairing which makes the graph $(k+1)$-connected.)

In the remaining part of this section let $G=(V, E)$ be a strongly connected, but not 2-connected digraph.

Lemma 3.7. If $b(G)=1$ (or $t(G)=1$ ), then $G$ can be made two-connected by adding $t(G)(b(G)$, resp.) edges.

Proof. If $b(G)=t(G)=1$, then one vertex can cover all the in-tight sets, namely, any vertex of the unique minimal in-tight set will do. (Observe, that if $B_{1}$ and $B_{2}$ are intersecting minimal in-tight sets, then $B_{1}^{*}$ and $B_{2}^{*}$ are disjoint out-tight sets.) The out-tight sets can also be covered by one vertex, hence any edge between the two covering vertices makes $G$ 2-connected.

If $t(G) \geq 3$, then any edge between a minimal out-tight set $K$ and $K_{c}^{*}$ is saturating. (Now $K_{c}$ is out-tight.)

If $t(G)=2$ and $b(G)=1$, then for every minimal out-tight set $K$, the container-set $K_{c}$ is out-tight. $\left(\left|K_{c}\right| \leq|V|-2\right.$.) Otherwise there were two crossing out-tight sets $K_{1}$ and $K_{2}$ in one of the container-sets such that $\left|V-\left(K_{1} \cup K_{2}\right)\right|=$ 1. But this case would yield two disjoint minimal in-tight sets, i.e. $K_{1}^{*}$ and $K_{2}^{*}$. Thus any edge between a minimal out-tight set $K$ and $K_{c}^{*}$ will decrease $t(G)$ by Lemma 3.3.

An important point of the proof is that the set of new edges determined by the pairing of Lemma 3.6 makes $G$ 2-connected, if $b(G)=t(G)=3$.

Lemma 3.8. If $b(G)=t(G)=3$, then $G$ can be made two-connected by adding 3 edges.

Proof. By Lemma 3.2, the minimal out-tight (and similarly, the minimal in-tight) sets are pairwise disjoint. Let us denote the minimal out-tight sets by $P, Q$ and $N$. By Lemma 3.6 we can find 3 edges $e, f, g$ such that every edge connects a minimal out-tight set to a minimal in-tight set so that these sets satisfy (3.1), and every minimal tight set is covered by one of these edges.

We claim that $G^{\prime}:=G+\{e, f, g\}$ is 2-connected. 
For suppose that there exists a set $X$ with only one out-neighbour in $G^{\prime}$ and with $|V-X| \geq 2$. We check several cases depending on the relation between $X$ and the sets $P, Q, N$.

If $X$ contains $P, Q$ and $N$, then at least one edge connects $X$ to a minimal in-tight set in $X^{*}$ (which is in-tight in $G$, too), a contradiction.

Suppose that $X$ includes two minimal out-tight sets $P$ and $Q$. If $N$ intersects $X$, then $N \cup X=V$, hence $X^{*} \subset N$ holds. Thus the minimal in-tight set in $X^{*}$ must be connected to $P$ or $Q$, a contradiction. Now we can assume that $N \subseteq V-X$. If $P$ and $Q$ are both connected to a vertex in $X \cup \Gamma^{+}(X)$ (this must be the case if $X$ is out-tight), then it is easy to see that $X^{*} \subseteq T_{c}$ holds for the minimal in-tight set $T$ in $X^{*}$, a contradiction. (If a minimal in-tight set $S$ would intersect $X^{*}$, then $S \cup X^{*}=V$ would follow, and there was no edge in $G^{\prime}$ from $S_{c}^{*}$ to $S$.)

If $X$ contains only one minimal out-tight set $P$, then $Q$ and $N$ are in $V-X$ (otherwise the intersection with $X$ would be out-tight). Thus $X \subseteq P_{c}$, and $P$ must be connected to $X^{*}$, a contradiction.

Lemma 3.9. If $b(G)=2$ and $t(G)=3$, then $G$ can be made two-connected by adding 3 edges.

Proof. Let $B$ and $B^{\prime}$ denote the minimal in-tight sets which are disjoint by Lemma 3.2. If both of the two container-sets $B_{c}$ and $B_{c}^{\prime}$ are in-tight, then the same proof works as in Lemma 3.8, if we choose three edges such that each of them connects two minimal tight sets for which (3.1) holds, and every minimal tight set is connected to a new vertex. It is easy to see that such an augmenting set exists by Lemma 3.3.

Suppose that this is not the case, i.e. - say $-V-B_{c}=\{v\}$ for some $v \in V$. Then $B^{\prime}=\{v\}$ and there exist two different in-tight sets $B_{1}$ and $B_{2}$ such that $B \subset B_{1} \cap B_{2}$ and $B_{1} \cup B_{2}=B_{c}$.

If $B_{c}^{\prime} \neq B^{\prime}$, then $V-B_{c}^{\prime} \subseteq B_{1} \cap B_{2}$, since $b(G)=2$ and by Lemma 3.1 . Moreover, $\left|V-B_{c}^{\prime}\right| \neq 1$, otherwise we could find four pairwise disjoint in-tight sets. (Now $B_{1}^{*}$ and $B_{2}^{*}$ are disjoint out-tight sets in $B_{c}^{\prime}$.) In this case there exists a minimal out-tight set $K \subseteq B_{1} \cap B_{2}$, and it is easy to see that (3.1) holds for $K$ and $B^{\prime}$. This implies that for any edge $e$ between $K$ and $v, b(G+e)=1$ and $t(G+e)=2$, by Lemma 3.3, and we are done by Lemma 3.7.

If $B_{c}^{\prime}=B^{\prime}$, then - since the container-sets of the minimal out-tight sets are pairwise disjoint - there exists a minimal out-tight set $K$ for which neither $v \in K_{c}$, nor $v \in \Gamma^{+}\left(K_{c}\right)$. Then (3.1) holds for $K$ and $B^{\prime}$, and we are done as before by Lemma 3.3 and 3.7.

The missing piece is the characterization of those graphs for which $b(G)=$ $t(G)=2$ but 3 edges are necessary for the augmentation.

Lemma 3.10. If $b(G)=t(G)=2$, then $G$ can not be made 2-connected by adding two edges if and only if $(*)$ there exist three in-tight (or three out-tight) sets $B_{1}, B_{2}, B_{3}$, such that 


$$
B_{1} \cap B_{2} \neq \emptyset,\left|B_{3}\right|=1, \quad \text { and } V-\left(B_{1} \cup B_{2}\right)=B_{3} .
$$

If $(*)$ holds, then $G$ can be made 2-connected by adding 3 edges.

Proof. It is clear that if $(*)$ holds, then $G$ can not be made 2 -connected by adding two edges, since any new edge can increase the number of in- (or out-) neighbours of at most one $B_{i}$. (Observe that $B_{1}^{*} \cap B_{2}^{*}=\emptyset$.)

Consider the other direction. Clearly $B_{c} \neq V$ for any minimal in-tight set $B$ if $b(G)=2$. If $\left(V-B_{c}\right)=\{v\}$ for some $B$ and some vertex $v$, then $B_{c}$ is not in-tight but the union of two in-tight sets $B_{1}$ and $B_{2}$. (Let $B_{1}$ be a maximal in-tight set in $B_{c}$, and let $B_{2}$ be an in-tight set which contains a fixed vertex $x \in B_{c}-B_{1}$.) Moreover, $\{v\}=B^{\prime}$ holds. Now $B_{1}, B_{2}$ and $B^{\prime}$ satisfy (*).

Hence we can assume that every container-set of an in-tight (out-tight) set is in-tight (out-tight). This means that an edge $x y$ is saturating if $x \in K, y \in B$, $x \in B_{c}^{*}$ and $y \in K_{c}^{*}$ for a minimal in-tight set $B$ and a minimal out-tight set $K$.

Suppose that $B_{c} \cap B_{c}^{\prime} \neq \emptyset$. Thus $B_{c} \cup B_{c}^{\prime}=V$, otherwise their intersection would be in-tight. Now there exists a minimal out-tight set $K$ in $B_{c}^{*} \cap B_{c}^{\prime}$. We claim that after adding an edge between $K$ and $B$, the new graph $G^{\prime}$ has $b\left(G^{\prime}\right)=$ $t\left(G^{\prime}\right)=1$. Indeed, it is easy to check that $B \subseteq K_{c}^{*}$ also holds. Now $G^{\prime}$ can be made 2 -connected by one new edge by Lemma 3.7 .

Assume that the two in-tight container-sets are disjoint and so are the two out-tight container-sets. We have to find a good pair for the minimal in-tight set $B$. Suppose that $B$ is bad for $K$ and for $K^{\prime}$ as well. Then $B_{c}$ is disjoint from $K_{c}^{*}$ or $K_{c}$ is disjoint from $B_{c}^{*}$, and similarly for $K^{\prime}$. Thus - without loss of generality - there exist two intersecting in-tight sets which are disjoint from a third in-tight set. Hence these three sets satisfy $(*)$. This completes the proof of the first claim.

Suppose now that $(*)$ holds. Note, that $B_{3}=C_{1} \cap C_{2}$ would imply $\left|\Gamma^{-}\left(B_{3}\right)\right| \geq$ 2 , hence it is impossible.

Observe, that the container-set $B$ of $B_{3}$ must be $B_{3}$ itself. (If $B$ intersects one of the other $B_{i}$ 's, the intersection is in-tight - it is not possible - or the union is $V$. Now $B^{*} \subseteq B_{1} \cap B_{2}$ follows, contradicting to $t(G)=2$.)

Hence any edge $e$ which gives a new neighbour to $B_{3}$, decreases $b(G)$ by one. For the new graph $G^{\prime}=G+e$ we get $b\left(G^{\prime}\right)=1$ and $t\left(G^{\prime}\right)=2$, thus two more edges are sufficient for the augmentation by Lemma 3.7.

From the previous lemmas the following theorem follows easily:

Theorem 3.11. Let $G$ be a strongly connected digraph. If at least one of $b(G)$ and $t(G)$ is not equal to 2 , then $m(G)=\max \{b(G), t(G)\}$ holds. If $b(G)=t(G)=2$, then $m(G)=2$ or $(*)$ holds, and $m(G)=3$.

The constructive proofs of Section 3 yield a combinatorial polynomial algorithm for the optimal augmentation. The basic subroutine is the standard max-flow min-cut computation, which must be applied when one determines the minimal tight sets or tests whether an edge is saturating or not. Another stepwhich can be done easily, as well - is deciding whether $(*)$ holds for a digraph with $b(G)=t(G)=2$. We omit the details. 
Theorem 3.12. There exists a combinatorial polynomial algorithm which determines an optimal augmenting set for a strongly connected digraph.

\section{The General Case}

In this section we use the structural results of Section 2, related to the families of independent pairs of sets, and simplify the general augmentation theorem of [FJ1].

Let $V$ be a ground-set and let $\mathcal{A}$ denote the set of all ordered pairs $(X, Y)$ with $X \subseteq V, Y \subseteq V$ and $X \cap Y=\emptyset$. The set of directed edges $(x y), x, y \in V$ is denoted by $A$. We call $X$ and $Y$ the tail and the head of the pair, respectively. A directed edge $(x \dot{y}) \in A$ covers a pair $(X, Y) \in \mathcal{A}$ if $x \in X, y \in Y$. We say that a sub-family $\mathcal{F}$ of $\mathcal{A}$ is independent if every edge of $A$ covers at most one member of $\mathcal{F}$. This is equivalent to requiring that there are no two members $\left(X_{i}, Y_{i}\right)(i=1,2)$ of $\mathcal{F}$ for which $X_{1} \cap X_{2} \neq \emptyset$ and $Y_{1} \cap Y_{2} \neq \emptyset$.

The main result of [FJ1] - a general min-max theorem on covering pairs of sets - has the following consequence for the optimal directed vertex-connectivity augmentation.

Recall, that a pair $(X, Y)$ is a one-way pair, if there is no edge from $X$ to $Y$. The deficiency of a one-way pair - with respect to $k$-connectivity - is $p_{\text {def }}(X, Y):=(k-|V-(X \cup Y)|)^{+}$.

Theorem 4.1. [FJ1] A digraph $D=(V, E)$ can be made $k$-vertex-connected by adding at most $\gamma$ new edges if and only if

$$
\sum\left(p_{\text {def }}(X, Y):(X, Y) \in \mathcal{F}\right) \leq \gamma
$$

holds for every family $\mathcal{F}$ of pairwise independent one-way pairs.

One of the reasons why we have no combinatorial algorithm yet for the general augmentation problem is that we have no efficient method to handle the one-way pairs, especially when the deficiency can be more than one. However, our results lead to some simplifications.

Theorem 4.1 from [FJ1] and Theorem 2.5 implies the following theorem concerning the $k \rightarrow k+1$ augmentation problem.

Theorem 4.2. Let $G$ be a k-connected digraph for which $m(G) \geq 2 k+2$ holds. Then $m(G)=\max \{b(G) ; t(G)\}$

With the help of Theorem 4.1 and Lemma 2.3 we get an approximation result, showing that the usual, and easily computable lower bound - the maximum number of pairwise disjoint tight sets - is not far from the optimum.

Theorem 4.3. Let $G$ be a k-connected digraph. Then $m(G) \leq \max \{b(G) ; t(G)\}+$ $k$ holds. 
This inequality is sharp. This is shown for arbitrary $k$ in [Jor3]. Note, that Theorem 4.3 is an improvement on the approximation result of [Jor3].

Theorem 4.2 suggests a combinatorial polynomial algorithm for the optimal $k \rightarrow k+1$ augmentation problem for every fixed $k$ : using Lemma 3.4, we can add new edges until the maximum of $b(G)$ and $t(G)$ becomes less than $2 k+2$. Then we can try all the possible augmenting sets (of size $2 k+1$ ).

Finally, let us deduce a splitting-type theorem from our results. We remark, that in the case of edge-connectivity, the powerful splitting off theorems of Lovász and Mader can be used, for example, for solving the optimal edge-connectivity augmentation problem, see [Fra1]. Simple examples show that in the case of vertex-connectivity, the corresponding statements on the existence of a "complete splitting off" do not hold, even if the vertex from which we split off has even degree or the same in- and out-degree. (We say that the edges can be split off completely from a vertex $s$ in a $k$-connected digraph if there exists a pairing of the edges with tail or head $s$ such that splitting off all these pairs and deleting $s$ we get a $k$-connected digraph.) However, from the augmentation theorem we can derive the following:

Theorem 4.4. Let $G=(V+s, E+F)$ be a k-connected directed graph for which $d:=\left|\Gamma^{+}(s)\right|=\left|\Gamma^{-}(s)\right| \geq 2 k-1$ holds and every edge $e \in F$ is critical. ( $F$ denotes the set of edges with tail s or head s.) Then the edges incidenting to $s$ can be split off completely.

Proof. For $k=1$ the statement is trivial, we can assume $k \geq 2$. Let $H:=(V, E)$. Since the edges of $F$ are critical, and $2 k-1 \geq k+1, b(H)=t(H)=d$, and the minimal in-tight (out-tight) sets - with respect to $(k-1)$-connectivity - are pairwise disjoint. Let $S_{B}$ and $S_{K}$ be the set of out-neighbours and in-neighbours of $s$, respectively. Observe that $S_{B}$ contains exactly one vertex from every minimal in-tight set in $H$. We claim that there exists an optimal augmenting set of edges, which makes $H k$-connected, with tails in $S_{K}$ and heads in $S_{B}$.

This follows from the fact that if we delete an edge of an optimal augmenting set $T$ from a $k$-connected digraph $H^{\prime}=(V, E \cup T)$, then exactly one minimal in-tight set $B$ (and out-tight set $K$ ) is generated - with respect to $(k-1)$ connectivity - and every new edge between $K$ and $B$ will increase the connectivity back to $k$. Thus we can change the edges of any optimal augmenting set step by step until all the tails are in $S_{K}$ and all the heads are in $S_{B}$. (Observe that $B \cap S_{B} \neq \emptyset$ and $K \cap S_{K} \neq \emptyset$ must hold.)

Now Theorem 4.2 gives that the smallest augmenting set has size $b(H)=$ $t(H)=d$. This implies the theorem. 


\section{References}

[ET1] K.P. Eswaran and R.E. Tarjan, Augmentation problems, SIAM J. Computing, Vol. 5, No. 4, 653-665, 1976

[Fra1] A. Frank, Augmenting graphs to meet edge-connectivity requirements, SIAM J. on Discrete Mathematics, Vol.5, No 1., 22-53, 1992

[Fra2] A. Frank, Connectivity augmentation, Proceedings of the Mathematical Programming Symposium, held in Ann Arbor (USA), 1994

[FJ1] A. Frank and T. Jordán, Minimal edge-coverings of pairs of sets, J. Combinatorial Theory, Ser. B, to appear

[HR1] T.-s. Hsu and V. Ramachandran, A linear time algorithm for triconnectivity augmentation, in: Proc. 32nd Annual IEEE Symp. on Foundations of Comp.Sci, 548- 559, 1991

[Jor1] T. Jordán, On the optimal vertex connectivity augmentation, J. Combinatorial Theory, Ser. B. 63, 8-20, 1995

[Jor2] T. Jordán, Optimal and almost optimal algorithms for connectivity augmentation problems, in: Proc. of the third IPCO conference (G. Rinaldi and L. Wolsey, eds.), 75-88, 1993

[Jor3] T. Jordán, Increasing the vertex-connectivity in directed graphs, in: Proceedings of the First Annual European Symposium on Algorithms, Bad Honnef, Springer Lecture Notes in Computer Science, 726, 236-247, 1993

[NGM1] D. Naor, D. Gusfield and Ch. Martel, A fast algorithm for optimally increasing the edge-connectivity, 31st Annual Symposium on Foundations of Computer Science, 698-707, 1990

[WN1] T. Watanabe and A. Nakamura, Edge-connectivity augmentation problems, Computer and System Siences, Vol 35, No. 1, 96-144, 1987

[WN2] T. Watanabe and A. Nakamura, A minimum 3-connectivity augmentation of a graph, J. Computer and System Sciences, Vol. 46, No.1, 91-128, 1993 